\title{
Recent Advances in Ureteral Tissue Engineering
}

\author{
Paul K. J. D. de Jonge • Vasileios Simaioforidis • Paul J. Geutjes • \\ Egbert Oosterwijk • Wout F. J. Feitz
}

Published online: 11 November 2014

(C) The Author(s) 2014. This article is published with open access at Springerlink.com

\begin{abstract}
Reconstruction of long ureteral defects often warrants the use of graft tissue and extensive surgical procedures to maintain the safe transport of urine from the kidneys to the urinary bladder. Complication risks, graft failure-related morbidity, and the lack of suitable tissue are major concerns. Tissue engineering might offer an alternative treatment approach in these cases, but ureteral tissue engineering is still an underreported topic in current literature. In this review, the most recent published data regarding ureteral tissue engineering are presented and evaluated, with a focus on cell sources, implantation strategies, and (bio)materials.
\end{abstract}

Keywords Ureter $\cdot$ Regeneration $\cdot$ Reconstruction $\cdot$ Tissue engineering $\cdot$ Regenerative medicine $\cdot$ Stem cells $\cdot$ Cells $\cdot$ Pre-implantation $\cdot$ Biomaterials $\cdot$ Animals

Both Paul K. J. D. de Jonge and Vasileios Simaioforidis contributed equally.

This article is part of the Topical Collection on Regenerative Medicine

P. K. J. D. de Jonge $(\bowtie) \cdot$ V. Simaioforidis $•$ P. J. Geutjes

E. Oosterwijk • W. F. J. Feitz

Department of Urology, Radboud Institute for Molecular Life

Sciences, Radboud University Medical Center, P.O. Box 9101, Geert

Grooteplein 26/28, 6525 GA Nijmegen, The Netherlands

e-mail: paul.dejonge@radboudumc.nl

V. Simaioforidis

e-mail: vasilis.simaioforidis@radboudumc.nl

P. J. Geutjes

e-mail: paul.geutjes@radboudumc.nl

E. Oosterwijk

e-mail: egbert.oosterwijk@radboudumc.nl

W. F. J. Feitz

e-mail: wout.feitz@radboudumc.nl

\section{Introduction}

Advances in endourology led to an increase in the number of ureteroscopies and nephroscopies during the last two decades worldwide. The complications of these procedures are often underreported, possibly due to lack of early recognition or short-term postoperative follow-up [1-3]. Strictures after ureteroscopy occur with an estimated frequency up to $3.5 \%$, which is more often than avulsion or major perforation $[4,5 \cdot, 6]$. In around $1 \%$ of the gynecological procedures, a ureteral injury occurs, which is estimated to account for up to $73 \%$ of all ureteral injuries $[3,7,8]$. In addition to these iatrogenic injuries, trauma can result in ureteral damage. A large retrospective analysis in the USA showed that $2.6 \%$ of all urogenital traumas involved the ureter between 2002 and 2006 [9]. The specific anatomic characteristics of the ureter, such as the segmental vascular supply, can be easily damaged, and the lack of native tissue limits surgical ureteral reconstruction. To repair the long ureteral defects, where an end-to-end anastomosis is not feasible for the urologist, several techniques have been introduced such as a ureteroneocystostomy, a Boari flap, ileal interposition, and renal autotransplantation. Ultimately, when surgical expertise is not available or the aforementioned techniques do not succeed, an undesirable nephrectomy is the only option $[10,11]$.

Tissue engineering might offer new treatment approaches in ureteral reconstruction to optimize the outcome in complicated cases, which currently have complication rates up to $25 \%$ [12]. The number of published manuscripts dealing with tissue engineering applications of the urinary system is quite extensive, particularly for urinary bladder reconstruction, but the number of research groups that focus on tissue engineering of the ureter is limited, which suggests that the development of an artificial construct suitable for ureteral reconstruction is challenging. Additionally, there might be less incentive to investigate tissue engineering approaches as the incidence of long ureteral injuries is lower than urethra or bladder injuries. 


\section{Ureteral Tissue Engineering}

In a recent review on ureteral tissue engineering [13], it was demonstrated that the evidence in the literature was inconclusive about the optimal tissue engineering approach to treat long ureteral injuries. Furthermore, compared to other parts of the urogenital tract, very few tissue engineering studies were performed. Most studies focused on tubular(ized) small intestinal submucosa (SIS) without cellular preseeding [14-20]. Other materials included collagen [21, 22] and Gore-Tex [23, 24]. In general, collagen and SIS, but not Gore-Tex, were capable of facilitating some degree of urothelium and smooth muscle regeneration. However, fibrosis occurred in most cases.

In this review, we present recent developments in ureteral tissue engineering and discuss currently used materials, construct design, cell sources, and implantation techniques. In addition to the recent literature reviews on ureteral tissue engineering, a Medline search was performed for papers published in the last 3 years using a previously published tissue engineering filter [25] combined with the $\mathrm{MeSH}$ term ureter. An overview of the recent studies is presented in Table 1 in which we focused on the early post-implantation complications and the presented solutions.

\section{Ureteral Defect Repair}

To study the effect of a tissue-engineered construct on the regeneration of the ureter, it is imperative to test the constructs in a ureteral defect model. Nevertheless, in three recent studies, the authors refrained from implantation of tissueengineered constructs in an induced ureteral defect model. Instead, the authors performed subcutaneous implantations in rats $(\mathrm{Xu}$, et al. [26]) and mice (Shi, et al. [27] and $\mathrm{Fu}$, et al. [28]). While these studies showed the potential of preimplantation for ureteral replacement, information about the behavior of the construct as ureteral replacement is lacking. In the intracorporeal environment, constructs are exposed to the toxic effects of urine and various mechanical forces [5•]. All three studies used a similar spiral poly(L-lactic acid) (PLLA) stent as the backbone of their construct. Xu et al. [26] implanted the spiral PLLA stents in the subcutis and used the body as a natural bioreactor to generate a tissue fleece around the stent. The newly formed tissue was then decellularized and re-seeded with primary urinary bladder urothelial cells. Cell proliferation was similar compared to SIS. Shi et al. [27] and Fu et al. [28] combined the same PLLA stent with electrospun collagen to improve cell attachment and cell proliferation. Before subcutaneous implantation in athymic mice, the final constructs were seeded with human adipose-derived stem cells (hADSCs) (Shi, et al. [27]) or human urothelial cells (hUC) (Fu, et al. [28]). Both authors were able to detect viable human cells 2 weeks post-implantation, demonstrating that the cells could survive the procedure. These studies solely indicate that the subcutis might be a suitable preimplantation site to generate a tubular pre-vascularized autologous tissue, which may prevent fibrosis when attempting to repair the ureter.

\section{Cell Sources}

The use and necessity of cell seeding of tissue-engineered constructs has been a matter of debate, but once it is considered, many options exist. Embryonic stem cells are highly controversial due to their origin and the risk of tumor formation. A safer and less controversial option is the use of autologous cells when available. Tissue biopsies can yield differentiated primary cells or multipotent cells like mesenchymal or adipose-derived stem cells. Most early studies in ureteral tissue engineering used bare scaffolds, and almost all of them resulted in fibrosis, which may indicate the necessity of cell seeding [14-19, 21-24, 33]. This is supported by previous

Table 1 Recent ureteral tissue engineering studies

\begin{tabular}{|c|c|c|c|c|c|c|}
\hline Authors & Animal model & Biomaterial & Cell seeded & Length (cm) & Technique & Outcome \\
\hline Xu et al. [26] & Rats (M) & PLLA & No & 0.9 & $\mathrm{~S}, \mathrm{~T}$ & $\mathrm{I}_{1}, \mathrm{~V}$ \\
\hline Shi et al. [27] & Mice $(F)$ & PLLA, Collagen & hADSC & - & $\mathrm{S}, \mathrm{T}$ & hUC \\
\hline Fu et al. [28] & Mice (M) & PLLA, Collagen & hUC & $1.0-1.5$ & $\mathrm{~S}, \mathrm{~T}$ & hUC \\
\hline Zhang et al. [29] & Dogs $(F)$ & Autologous graft & No & 3.0 & $\mathrm{P}, \mathrm{T}$ & UC, SMC, V \\
\hline Salehipour et al. [30] & Dogs (M) & $\mathrm{AM}$ & No & 3.0 & $\mathrm{~T}^{*}$ & $\mathrm{~L}, \mathrm{H}, \mathrm{F}, \mathrm{I}_{2}$ \\
\hline Zhao et al. [31••] & Rabbits (F) & VECM & $\mathrm{ADSC}$ & 3.0 & $\mathrm{~T}$ & $\mathrm{UC}, \mathrm{SMC}$ \\
\hline Liao et al. [32••] & Rabbits (M) & BAM & MSC, SMC & 4.0 & $\mathrm{P}, \mathrm{T}^{*}$ & $\mathrm{I}_{1}, \mathrm{UC}, \mathrm{SMC}$ \\
\hline De Jonge and Simaioforidis et al. (unpublished) & Pigs (F) & Collagen & $\mathrm{UC}, \mathrm{SMC}$ & 5.0 & $\mathrm{~T}$ & $\mathrm{UC}, \mathrm{SMC}, \mathrm{L}, \mathrm{F}, \mathrm{H}$ \\
\hline
\end{tabular}

PLLA poly(L-lactic acid), $A M$ amniotic membrane, VECM vessel extracellular matrix, BAM bladder acellular matrix, (h)ADSC (human) adipose derived stem cell, $M S C$ mesenchymal stem cell, (h)UC (human) urothelial cell, $S M C$ smooth muscle cell, $S$ subcutaneous implantation, $P$ pre-implantation, $T$ tubular, $T^{*}$ tubularized, $I$ inflammation $\left(I_{1}\right.$ mild, $I_{2}$ severe), $V$ vascularization, $F$ fibrosis, $H$ hydronephrosis, $L$ urine leakage 
statements that cell seeding is required for large defects $(>1.0 \mathrm{~cm}$ from the wound edge) to promote tissue regeneration and to prevent scar formation [34]. In most recent studies, cell seeding or pre-implantation of the scaffolds was explored to improve regeneration $[27-29,31 \bullet \bullet, 32 \bullet \bullet]$.

Fu et al. [28] used primary urothelial cells, isolated from patients that underwent nephrectomy, which were seeded on spiral PLLA stents and subcutaneously implanted in a nude mouse model for 2 weeks. The grafts resulted in a thin tissue capsule in which the seeded cells were still present and viable. This successful approach is relatively straightforward, albeit time consuming since it takes 4 weeks before ureter reconstruction can be performed; the cells are expanded for 2 weeks, followed by pre-implantation for 2 weeks.

A faster approach would be the implantation of only the cell-seeded construct. To investigate this approach, we implanted 5.0-cm-long highly porous tubular $0.5 \%$ type-I collagen constructs to repair a full ureteral defect in 11 female Landrace pigs (unpublished data). In brief, primary urothelial (UC) and smooth muscle cells (SMCs) were isolated from porcine urinary bladder biopsies [35]. First, the scaffolds were homogenously seeded with urinary bladder-derived SMCs, followed by luminal seeding of urinary bladder-derived UC. The right ureter was approached and mobilized through a midline incision, and a $5.0-\mathrm{cm}$ segment of the ureter was removed and replaced with an equally sized scaffold. A 6 Fr double-J stent was placed to facilitate urinary flow, and animals were followed up to 4 weeks. In 7/11 pigs, abdominal swelling due to urine leakage was observed after 2-3 weeks. The other animals developed strictures and hydronephrosis despite the presence of the stent. Upon analysis, it became clear that the urine leakage could be attributed to insufficient mechanical strength of the collagen scaffolds, which resulted in ruptures or dissections of the scaffolds. In the animals where the scaffold remained patent, the scaffold was mostly covered by a single layer of urothelial cells. Extensive neovascularization and some SMC ingrowth were observed (Fig. 1). Although the collagen construct with primary urinary bladder cells was suitable for ureteral reconstruction, we can conclude that back-bone biodegradable synthetic materials are needed to bear mechanical loads when attempting to repair an unsupported, mobile organ like the ureter.

One of the major disadvantages of primary cells, especially for urothelium, is that the cells cannot safely be harvested in case of possible malignancies [36]. Also, suitable tissue may not always be available for cell isolation. Therefore, alternative cell sources are being explored with a focus on mesenchymal (MSCs) and adipose-derived stem cells (ADSCs). These cells can differentiate into multiple cell lineages, including muscle and epithelium, without the risk of tumor formation [37-40]. Additionally, they are associated with anti-inflammatory properties and the capability to produce several cytokines that are associated with normal wound healing [41].
Zhao et al. [31••] isolated ADSC from rabbits and differentiated these towards a SMC phenotype before cell seeding and implantation. The cells were seeded on decellularized rabbit abdominal aorta to prepare a vascular extracellular matrix (VECM). Cell-seeded scaffolds were used to replace a 3.0-cm-long defect of the rabbit ureter. After 16 weeks, the defect was characterized by a well-organized muscle layer and stratified urothelium similar to native tissue. Strictures and hydronephrosis were absent. The authors attributed the positive results to the stimulating effect of ADSC on SMC proliferation and differentiation and the use of a graft containing many natural occurring growth factors [42]. Additionally, Shi et al. [27] showed that human ADSC can survive and maintain their phenotype for at least 2 weeks when implanted subcutaneously in nude mice, showing the possibility to use these cells for their stimulating properties in time. Alternatively, MSC can be used. These cells possess similar properties as ADSC but are isolated from the bone marrow. The harvesting procedure of these cells is painful, the differentiation potential decreases with age, and the number isolated cells is limited compared to ADSC [43, 44]. Liao et al. [32••] used MSC in combination with SMC seeded on bladder acellular matrix (BAM) to repair a 4.0-cm ureteral defect in rabbits. The BAM was seeded with bone marrow-derived mesenchymal stem cells (MSCs) on one side and urinary bladder-derived SMCs on the other side to create tissue-engineered tubular grafts (TETGs). The TETG was tubularized around a catheter and pre-implanted in the omentum of rabbits for 2 weeks. During this pre-implantation, the MSC differentiated and formed a single-cell-layered epithelium. Next, the ureteral defect was repaired, where a multilayer urothelium with central neovascularization was observed after 16 weeks. No strictures or hydronephrosis was observed, even though the ureteral catheter was removed 6 weeks postoperatively. Without MSC, the ureter repair resulted in scar formation and severe hydronephrosis. The investigators reasoned that the formation of the single-layered epithelium during the pre-implantation phase might have protected the surrounding tissue against urine.

These examples show the potential of stem cells as an alternative cell source for ureteral tissue engineering when insufficient donor tissue is available.

\section{Full-Circumference Ureteral Defect Repair}

Major ureteral reconstructions are required when a complete segment of the ureter needs to be replaced. Onlay graft repair is most often impossible and can only be applied in stricture repair. It is therefore not surprising that the majority of the studies focus on the repair of long (relative to the total length of the ureter) defects using tubular or tubularized constructs. Zhang et al. [29] used 3.0-cm-long tubular autologous 

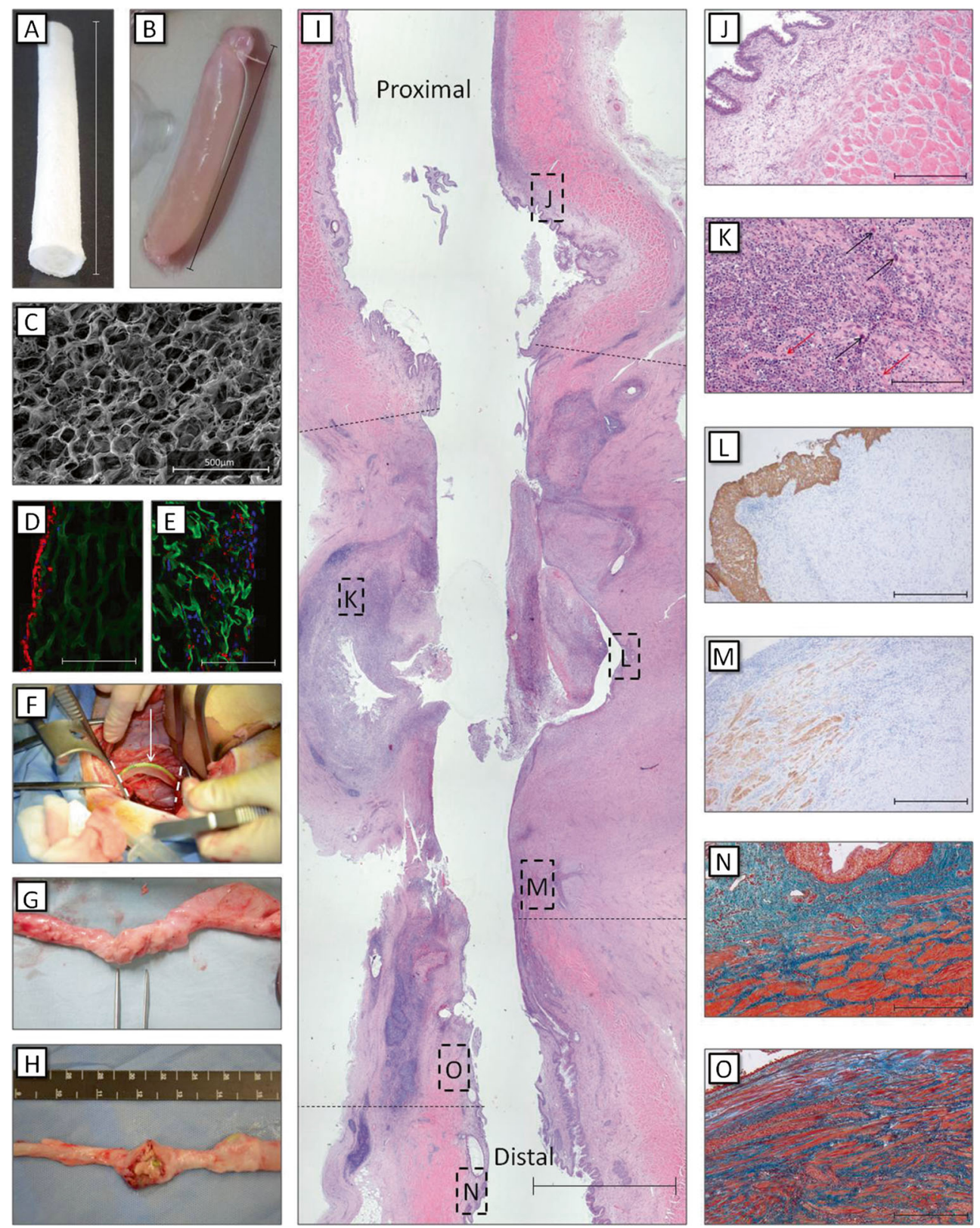

Fig. 1 Implantation strategy and outcome after ureteral reconstruction using tubular collagen scaffolds. a, b Macroscopic overview of a tubular $0.5 \%$ type-I collagen scaffold (length $=6 \mathrm{~cm}, \varnothing=6 \mathrm{~mm}$ ). c SEM overview of the scaffold surface, which was highly porous, facilitating cell penetration into the scaffold (scale bar $=500 \mu \mathrm{m}$ ) d Immunofluorescent staining for collagen (green), nuclei (blue), and RCK103 (red) of a cell-seeded scaffold. Urothelial cells (RCK103 positive) were lining the scaffold (scale bar $=400 \mu \mathrm{m}$ ). e Immunofluorescent staining for collagen (green), nuclei (blue), and $\alpha$ smooth muscle actin (red) of a cell-seeded scaffold. Smooth muscle cells ( $\alpha$ smooth muscle actin positive) were found throughout the scaffold (scale bar=400 $\mu \mathrm{m}$ ). f The scaffolds were implanted by end-to-end anastomosis. $\mathbf{g}$, $\mathbf{h}$ Ureteral regeneration was evaluated after 1 month. Four animals showed intact ureters (g), while seven animals presented with defects or dissections (h). i Histological overview

of a regenerating ureter (scale bar $=5 \mathrm{~mm}$ ). Black dotted lines indicate the anastomosis sites. Specific locations are highlighted $(J-O)$. j Hematoxylin and eosin (HE) staining of the native ureter (scale bar= $400 \mu \mathrm{m}$ ). $\mathbf{k}$ Inflammatory response in the regenerating tissue near scaffold remnants (red arrows). Mostly lymphocytes, a few granulocytes, and some multinucleated giant cells (black arrows) were observed (scale bar $=200 \mu \mathrm{m}$ ). I Pancytokeratin staining in the middle of the neo-ureter, indicating the presence of (multilayered) urothelium (scale bar= $400 \mu \mathrm{m})$. m Smoothelin staining near the anastomosis site, indicating ingrowth and maturation of smooth muscle tissue into the neo-ureter (scale bar $=400 \mu \mathrm{m}$ ). $\mathbf{n}$ Masson's trichrome staining of the native ureter (scale bar $=400 \mu \mathrm{m}$ ). o Masson's trichrome staining near the anastomosis site, indicating the ingrowth of new muscle tissue (scale bar $=400 \mu \mathrm{m})$

connective tissue that was formed after the implantation of silicon tubes in the peritoneal cavity of dogs. By maintaining

one third of the native ureter, the investigators kept adequate vascularization and they managed to generate new tissue 
which was similar to the normal ureter. After 12 weeks, the tubular construct was completely lined by multilayered urothelium and presented with an organized muscle layer and mucosal folds. While these results are very promising, one has to realize that it is unlikely that one third of healthy ureter is present in the clinical situation, e.g., in case of severe adhesions or prolonged avascularity. It is generally accepted that tissue ingrowth after $1.0 \mathrm{~cm}$ becomes increasingly difficult and is likely to be accompanied by fibrosis [34]. By maintaining the ureteral segment, the authors avoided this challenge.

\section{Pre-implantation}

The lack of functional urothelium and adequate vascularization may contribute to stricture formation and fibrosis as urine can freely damage the regenerating tissue $[5 \bullet, 45]$. Preimplantation promotes vascularization and helps to maintain the viability of the seeded cells in vivo. Different preimplantation sites have been used for various applications, including the omentum [46, 47], peritoneum [48, 49], and subcutis [49, 50]. Zhang et al. [29] and Liao et al. [32・•] took advantage of pre-implantation before repairing a ureteral defect. Zhang et al. exploited preimplantation to create a tubular scaffold from the fibrous capsule which was formed in the peritoneal cavity. Liao et al. [32••] used omental pre-implantation as an in vivo bioreactor to increase neovascularization in the construct. Additionally, it allowed the formation of a one-layer epithelial structure which may protect the construct after implantation in the toxic urine-rich environment.

Ideally, when harvesting the pre-implanted material, the newly formed blood vessels should remain intact. A mobile pre-implantation site close to the ureteral defect repair site, like the greater omentum, might be suitable for this as flaps can easily be mobilized most of the time. Although preimplantation techniques are promising, they are time consuming and require a second surgical procedure. Therefore, they may not always be applicable in case of acute problems and unplanned procedures, which is often the case with ureteral trauma.

\section{Decellularized Tissue and Synthetic Polymers}

A variety of materials has been used as scaffolding material. Most studies used decellularized tissues as opposed to "manmade" scaffolds in the past decades. The advantages of decellularized tissues include preservation of the native tissue architecture and inclusion of tissue-specific growth factors and other signaling molecules [42]. In the past, SIS has been the decellularized tissue of choice. The results, however, were not optimal in ureteral tissue engineering. Recently, Salehipour et al. [30] used amniotic membrane (AM), which is known for its anti-inflammatory properties, as a biomaterial to reconstruct long ureteral defects. In dogs, a $3.0-\mathrm{cm}$ segment of the ureter was replaced by tubularized decellularized AM. Two out of seven animals died due to urine leakage, and another animal showed severe hydronephrosis, acute and chronic inflammation, and the formation of granulation tissue. The other animals presented with mild pelvicaliectasis and fibrosis of the reconstructed segment with lymphatic and granulocytic infiltration. Where Koziak et al. [51] showed encouraging results when AM was used as an onlay graft in 2007, the authors of this study concluded that AM did not act as a favorable material when used in full defects. This result was similar to a previous study by Osman et al. in 2004 [33]. Decellularized blood vessels and bladder acellular matrix have recently been used with promising results, but these results may also be caused by the use of stem cells and pre-implantation techniques, something that was not done in combination with SIS for the ureter $[31 \bullet \cdot, 32 \bullet \cdot]$.

Besides decellularized tissues, man-made scaffolds can be used. The advantage of these scaffolds is a higher degree of plasticity, good mechanical properties, and they are well defined. Most materials can be prepared in any shape (e.g., flat, film, or tube) or size, and different proteins and bioactive molecules can be added as demonstrated in the recent publications using spiral PLLA stents in combination with collagen and our tubular collagen scaffolds. When improvements such as increased mechanical strength are required, these man-made scaffolds can easily be tailored compared to decellularized tissue.

\section{Animal Models}

In recent ureteral replacement animal studies, rabbits $[31 \cdot \bullet$, $32 \bullet \bullet]$, dogs [29, 30], and in our case, pigs (unpublished) were used, while subcutaneous implantation studies were performed in rats [26] or mice [27, 28]. The pig is the preferred model because the abdominal anatomy of pigs and humans is similar $[52,53]$. Nevertheless, the lack of recent pig studies might be associated with the high incidence of fibrosis and fast growth of the animal, as mostly fast-growing young pigs are used. This may influence tissue regeneration and cause mechanical stress on the tissue constructs. The ideal animal should have a similar size and abdominal anatomy as humans, be fully grown, and have similar wound healing characteristics. Potential candidate animals include goats, sheep, cattle, and horses. In general, randomized controlled trials preceded by extensive toxicity 
studies are required before a new technique is widely used in the clinic. However, in tissue engineering, it is unethical to perform safety studies in healthy patients and there is often a lack of golden standard treatments. Therefore, choosing the right animal models is critical to predict the expected clinical outcome as good as possible [54].

\section{Conclusions}

Ureteral reconstruction should focus on the maintenance of safe urine transport from the kidney to the bladder. Fast development of a vascular system, a functional smooth musculature, and a urothelial barrier is critical for the success of constructs as the lack of these layers may result in strictures and hydronephrosis, even when stents are used. In the past few years, clear advancements have been made in ureteral tissue engineering. Specifically, the cell source, implantation techniques, and new biomaterials have improved the tissue engineering of the ureter. Decellularized tissues or scaffolds with added natural proteins and other molecules may perform better than simple scaffolds; however, these were only studied in the context of stem cells. Despite these advancements, published research in the area of ureteral tissue engineering is scarce. Many recent studies do not address the behavior of the constructs in a ureteral replacement setting. To increase our knowledge on the effect of different materials, cell sources, and implantation techniques, future studies should attempt to repair a full ureteral defect. Current literature suggests that the use of mesenchymal and adipose-derived stem cells, seeded on any type of mechanically suitable bioactive material, is optimal for ureteral regeneration. In addition, preimplantation of these constructs in the omentum may improve the final outcome by increasing vascularization and triggering stem cell differentiation. However, when using the body as an in vivo bioreactor, the long incubation time may be problematic in ureteral repair. Finally, different pre-clinical animal models should be evaluated to prevent species-related result bias prior to commencing clinical trials.

\section{Compliance with Ethics Guidelines}

Conflict of Interest Paul K.J.D. de Jonge, Vasileios Simaioforidis, Dr. Paul J. Geutjes, Dr. Egbert Oosterwijk, and Dr. Wout F.J. Feitz each declare no potential conflicts of interest.

Human and Animal Rights and Informed Consent This article does not contain any studies with human or animal subjects performed by any of the authors.

Open Access This article is distributed under the terms of the Creative Commons Attribution License which permits any use, distribution, and reproduction in any medium, provided the original author(s) and the source are credited.

\section{References}

Papers of particular interest, published recently, have been highlighted as:

- Of importance

•- Of major importance

1. Vakili B et al. The incidence of urinary tract injury during hysterectomy: a prospective analysis based on universal cystoscopy. Am J Obstet Gynecol. 2005;192(5):1599-604.

2. Dandolu Vet al. Accuracy of cystoscopy in the diagnosis of ureteral injury in benign gynecologic surgery. Int Urogynecol J Pelvic Floor Dysfunct. 2003;14(6):427-31.

3. Dowling RA, Corriere Jr JN, Sandler CM. Iatrogenic ureteral injury. J Urol. 1986;135(5):912-5.

4. Johnson EB et al. Obstetric complications of ureteroscopy during pregnancy. J Urol. 2012;188(1):151-4.

5. Adamowicz $\mathrm{J}$ et al. Urine is a highly cytotoxic agent: does it influence stem cell therapies in urology? Transplant Proc. 2012;44(5):1439-41. With the application of stem cells in ureteral tissue engineering, this study demonstrates the toxic influence of urine on seeded cells. Care should be taken to ensure cell survival when unprotected tissue is exposed to urine.

6. Ostrzenski A, Radolinski B, Ostrzenska KM. A review of laparoscopic ureteral injury in pelvic surgery. Obstet Gynecol Surv. 2003;58(12):794-9.

7. Lee RA, Symmonds RE, Williams TJ. Current status of genitourinary fistula. Obstet Gynecol. 1988;72(3 Pt 1):313-9.

8. St Lezin MA, Stoller ML. Surgical ureteral injuries. Urology. 1991;38(6):497-506

9. Siram SM et al. Ureteral trauma: patterns and mechanisms of injury of an uncommon condition. Am J Surg. 2010;199(4):566-70.

10. Peters PC, Sagalowsky AL. Genitourinary trauma. In: Walsch PC et al., editors. Campbell's urology. Philadelphia: WP Saunders; 1992. p. 2571-94.

11. Png JC, Chapple CR. Principles of ureteric reconstruction. Curr Opin Urol. 2000;10(3):207-12.

12. Elliott SP, McAninch JW. Ureteral injuries: external and iatrogenic. Urol Clin North Am. 2006;33(1):55-66.

13. Simaioforidis $\mathrm{V}$ et al. Ureteral tissue engineering: where are we and how to proceed? Tissue Eng Part B Rev. 2013;19(5):413-9.

14. Liatsikos EN et al. Ureteral reconstruction: small intestine submucosa for the management of strictures and defects of the upper third of the ureter. J Urol. 2001;165(5):1719-23.

15. Smith 3rd TG et al. Ureteral replacement using porcine small intestine submucosa in a porcine model. Urology. 2002;60(5): 931-4.

16. Shalhav AL et al. Laparoscopic replacement of urinary tract segments using biodegradable materials in a large-animal model. J Endourol. 1999;13(4):241-4.

17. Duchene DA et al. Ureteral replacement using small-intestinal submucosa and a collagen inhibitor in a porcine model. J Endourol. 2004;18(5):507-11.

18. Sofer $\mathrm{M}$ et al. Ureteral segmental replacement using multilayer porcine small-intestinal submucosa. J Endourol. 2002;16(1):27-31.

19. El-Assmy A et al. Use of single layer small intestinal submucosa for long segment ureteral replacement: a pilot study. J Urol. 2004;171(5):1939-42.

20. El-Hakim A et al. First prize: ureteral segmental replacement revisited. J Endourol. 2005;19(9):1069-74.

21. Tachibana M, Nagamatsu GR, Addonizio JC. Ureteral replacement using collagen sponge tube grafts. J Urol. 1985;133(5):866-9. 
22. Dahms SE et al. Free ureteral replacement in rats: regeneration of ureteral wall components in the acellular matrix graft. Urology. 1997;50(5):818-25.

23. Baltaci $\mathrm{S}$ et al. Failure of ureteral replacement with Gore-Tex tube grafts. Urology. 1998;51(3):400-3.

24. Sabanegh Jr ES, Downey JR, Sago AL. Long-segment ureteral replacement with expanded polytetrafluoroethylene grafts. Urology. 1996;48(2):312-6.

25. Sloff $\mathrm{M}$ et al. Tissue engineering in animal models for urinary diversion: a systematic review. PLoS One. 2014;9(6):e98734.

26. $\mathrm{Xu} \mathrm{Y}$ et al. Autologous urothelial cells transplantation onto a prefabricated capsular stent for tissue engineered ureteral reconstruction. J Mater Sci Mater Med. 2012;23(4):1119-28.

27. Shi JG et al. Tissue engineering of ureteral grafts by seeding urothelial differentiated hADSCs onto biodegradable ureteral scaffolds. J Biomed Mater Res A. 2012;100(10):2612-22.

28. Fu WJ et al. New ureteral scaffold constructed with composite poly(L-lactic acid)-collagen and urothelial cells by new centrifugal seeding system. J Biomed Mater Res A. 2012;100(7):1725-33.

29. Zhang $J$ et al. Ureteral reconstruction using autologous tubular grafts for the management of ureteral strictures and defects: an experimental study. Urol Int. 2012;88(1):60-5.

30. Salehipour $\mathrm{M}$ et al. Is amniotic membrane a suitable biomaterial for reconstruction of long ureteral defects? Saudi J Kidney Dis Transpl. 2013;24(1):135-8.

31.• Zhao Z et al. Differentiation of adipose-derived stem cells promotes regeneration of smooth muscle for ureteral tissue engineering. J Surg Res. 2012;178(1):55-62. The authors of this manuscript are the first to successfully use adipose-derived stem cells to repair a full ureteral defect. They differentiated the cells to a smooth muscle cell phenotype and found organized muscle bundles 16 weeks after implantation.

$32 . \bullet$ Liao $\mathrm{W}$ et al. Construction of ureteral grafts by seeding bone marrow mesenchymal stem cells and smooth muscle cells into bladder acellular matrix. Transplant Proc. 2013;45(2):730-4. This study shows the first successful application of bone marrow mesenchymal stem cells to repair a full ureteral defect. An organized smooth musculature and multilayer urothelium were found throughout the neo-ureter.

33. Osman $\mathrm{Y}$ et al. Canine ureteral replacement with long acellular matrix tube: is it clinically applicable? J Urol. 2004;172(3):1151-4.

34. Chen F, Yoo JJ, Atala A. Experimental and clinical experience using tissue regeneration for urethral reconstruction. World J Urol. 2000;18(1):67-70.

35. Geutjes $P$ et al. Tissue engineered tubular construct for urinary diversion in a preclinical porcine model. J Urol. 2012;188(2):653-60.

36. Roupret $\mathrm{M}$ et al. European guidelines on upper tract urothelial carcinomas: 2013 update. Eur Urol. 2013;63(6):1059-71.
37. Tian $\mathrm{H}$ et al. Differentiation of human bone marrow mesenchymal stem cells into bladder cells: potential for urological tissue engineering. Tissue Eng Part A. 2010;16(5):1769-79.

38. Anumanthan $\mathrm{G}$ et al. Directed differentiation of bone marrow derived mesenchymal stem cells into bladder urothelium. J Urol. 2008;180(4 Suppl):1778-83.

39. Zuk PA et al. Multilineage cells from human adipose tissue: implications for cell-based therapies. Tissue Eng. 2001;7(2):211-28.

40. Drzewiecki BA, Thomas JC, Tanaka ST. Bone marrow-derived mesenchymal stem cells: current and future applications in the urinary bladder. Stem Cells Int. 2011;2010:765167.

41. Blaber SP et al. Analysis of in vitro secretion profiles from adiposederived cell populations. J Transl Med. 2012;10:172.

42. Chun SY et al. Identification and characterization of bioactive factors in bladder submucosa matrix. Biomaterials. 2007;28(29): 4251-6.

43. Shukla D et al. Bone marrow stem cells for urologic tissue engineering. World J Urol. 2008;26(4):341-9.

44. Lee OK et al. Isolation of multipotent mesenchymal stem cells from umbilical cord blood. Blood. 2004;103(5):1669-75.

45. Azadzoi KM et al. Atherosclerosis-induced chronic ischemia causes bladder fibrosis and non-compliance in the rabbit. J Urol. 1999;161(5):1626-35.

46. Atala A et al. Tissue-engineered autologous bladders for patients needing cystoplasty. Lancet. 2006;367(9518):1241-6.

47. Baumert $\mathrm{H}$ et al. Development of a seeded scaffold in the great omentum: feasibility of an in vivo bioreactor for bladder tissue engineering. Eur Urol. 2007;52(3):884-90.

48. Campbell JH, Efendy JL, Campbell GR. Novel vascular graft grown within recipient's own peritoneal cavity. Circ Res. 1999;85(12):1173-8.

49. Chue WL et al. Dog peritoneal and pleural cavities as bioreactors to grow autologous vascular grafts. J Vasc Surg. 2004;39(4):859-67.

50. Sparks CH. Silicone mandril method for growing reinforced autogenous femoro-popliteal artery grafts in situ. Ann Surg. 1973;177(3): 293-300.

51. Koziak A et al. Early experience in reconstruction of long ureteral strictures with allogenic amniotic membrane. Int $\mathrm{J}$ Urol. 2007;14(7):607-10.

52. Dalmose AL et al. Surgically induced urologic models in swine. $\mathrm{J}$ Invest Surg. 2000;13(3):133-45.

53. Swindle MM, Smith AC, Goodrich JA. Chronic cannulation and fistulization procedures in swine: a review and recommendations. $\mathrm{J}$ Invest Surg. 1998;11(1):7-20.

54. Oerlemans AJ et al. Regenerative urology clinical trials: an ethical assessment of road blocks and solutions. Tissue Eng Part B Rev. 2013;19(1):41-7. 